Research Article

\title{
Model Predictive Control Based on Parametric Disturbance Compensation
}

\author{
Lingliang Xu $\mathbb{D}^{1},{ }^{1}$ Guiming Chen ${ }^{\mathbb{D}}{ }^{1}$ Guangshuai Li $\mathbb{D}^{1},{ }^{2}$ and Qiaoyang Li ${ }^{1}$ \\ ${ }^{1}$ College of Combat Support, Xi'an Research Institute of High-Tech, Xi'an 710025, China \\ ${ }^{2}$ College of Nuclear Engineering, Xi'an Research Institute of High-Tech, Xi'an 710025, China \\ Correspondence should be addressed to Guiming Chen; 792757066@qq.com
}

Received 16 March 2020; Revised 26 April 2020; Accepted 19 September 2020; Published 7 October 2020

Academic Editor: Jose Vicente Salcedo

Copyright (c) 2020 Lingliang Xu et al. This is an open access article distributed under the Creative Commons Attribution License, which permits unrestricted use, distribution, and reproduction in any medium, provided the original work is properly cited.

Model predictive control (MPC) has been widely implemented in the motor because of its simple control design and good results. However, MPC relies on the permanent magnet synchronous motor (PMSM) system model. With the operation of the motor, parameter drift will occur due to temperature rise and flux saturation, resulting in model mismatch, which will seriously affect the control accuracy of the motor. This paper proposes a model predictive control based on parameter disturbance compensation that monitors system disturbances caused by motor parameter drift and performs real-time parameter disturbance compensation. And the frequency-domain method was used to analyze the convergence and filterability of the model. The Bode diagram of measurement error and input disturbance was studied when the parameters were underdamped, critically damped, and overdamped. Guidelines for parameter selection are given. Simulation results show that the proposed method has good dynamic performance, anti-interference ability, and parameter robustness, which effectively avoids the current static difference and oscillation problems caused by parameter changes.

\section{Introduction}

Since the discovery of electromagnetic induction by Faraday in 1831, scholars around the world have been exploring new areas of application, among which electromagnetic launch technology is one of the newer fields. As a driving device of electromagnetic launch technology, electric motors play an important role. The permanent magnet synchronous motor (PMSM) has the obvious advantages of small size, low power consumption, high transmission efficiency, and high powerweight ratio [1], making it the first choice in the electromagnetic launch research field.

PMSM is a complex system with multiple variables, strong coupling, nonlinearity, and variable parameters, hence the need for the adoption of a reasonable control method to control it [2]. Traditional control methods include vector control and direct torque control.

The vector control technology is based on the idea that the armature current and the excitation current of the direct current (DC) motor are perpendicular to each other without coupling and independent control. Based on coordinate transformation, the size and direction of the stator current in the synchronous rotating coordinate system are controlled to achieve decoupling. The purpose is to realize the decoupling of magnetic field and torque. But in the vector control module, there are many intermediate variables involved in the design of the parameter setting method of the speed loop PI regulator, and in many cases are approximate results obtained based on assumptions. To overcome the shortcomings, Singh et al. [3] discussed the changes of various parameters. The effect on the output and torque of the PI regulator exposed the limitations of PI control. Templos-Santos et al. [4] evaluated the efficiency of five bionic optimization algorithms by introducing two reference speed profiles and finally selected the free radical algorithm to adjust PI control device parameters.

Direct torque control (DTC) utilizes bang-bang control to generate pulse width modulation (PWM) signals and optimally control the inverter switches to obtain high dynamic performance of torque. DTC solves some of the problems in vector 
control, such as the complexity of calculation and the large error caused by model mismatch. However, the torque and magnetic flux pulsation generated by DTC are large, and the switching frequency is variable [5-7]. In order to overcome the shortcomings of large torque and magnetic flux pulsations, Zhang and Zhu [8] used an appropriate permutation vector sequence and adopted the amplitude and phase compensation voltage model of the low-pass filter to obtain an accurate stator flux linkage estimation to reduce smaller pulsation. Zhang and Zhu [9] established a unified switch table and performed threevector selection instead of the traditional two-vector selection to obtain smaller torque and flux ripple.

To further improve the control performance of the PMSM and solve the problems of motor control under complex conditions, various control methods such as sliding mode control of variable structure, modern intelligent control, and model predictive control (MPC) have been proposed. MPC occupies an important position due to its multiobjective, multivariable, and multiconstraint control characteristics, as well as its intuitive and simple design methods $[10,11]$.

MPC discretizes the mathematical model of PMSM to obtain the control vector for the next period. Therefore, MPC depends on the PMSM system model, but as the motor runs, the motor parameters will be affected by factors such as temperature, motor operating state, and magnetic field saturation. Parameter drift occurs, model mismatch occurs, and then reusing the marked parameters on the motor nameplate will cause greater errors, which will reduce the control accuracy of the PMSM system [12]. Young et al. [13] carried out an experimental evaluation of the model mismatch caused by changes in load parameters and analyzed that the steady-state performance and transient performance of PMSM were greatly affected by the model error. In order to reduce the control deterioration due to model mismatch, Xia et al. [14] estimated the model uncertainty by minimizing the error between the measured current and the model current, but this method focuses more on the uncertainty of the converter than the model parameters. Antoniewicz and Kazmierkowski [15] proposed an improved direct power prediction algorithm to minimize the difference between instantaneous power and reactive power and avoid the impact of inductance changes.

With the operation of the motor, motor parameters will be affected by factors such as temperature, motor operating state, magnetic field saturation, various disturbances, and so on, resulting in parameter drift and model mismatch and severely affecting the stability of PMSM. This paper analyzes the effects of model mismatch caused by changes in parameter resistance, inductance, and magnetic flux. Aiming at the disturbance caused by parameter drift, external input deviation, and measurement error during the operation of the motor, an expanded state observer is designed to monitor the disturbance in real time and perform disturbance compensation to improve the system's robust performance [16-21]. In view of the problem of gain parameters in the extended state observer, the frequency-domain analysis method is used to determine the appropriate parameters to improve the stability of the motor operation. Simulation results show that the model established has good parameter robustness and avoids current static differences and oscillation problems caused by parameter drift. Under actual production conditions, this control method is helpful to solve the model mismatch problem that occurs during the operation of the motor, which is conducive to the smooth operation of the motor without large fluctuations, and the production process is not interrupted and the production operations are carried out in an orderly manner.

The structure of this paper is as follows. Section 2 describes the PMSM mathematical model and analyzes the model mismatch caused by parameter changes. Section 3 discretizes the mismatched mathematical model and obtains the control vector for the next period. Considering the influence of parameter changes, an extended state observer is constructed. In Section 4, we consider the one-shot delay of the digital control system and perform control delay compensation. In Section 5, the frequency-domain analysis method is used to analyze the convergence and filtering of the model. Section 6 gives the simulation results. Section 7 concludes the paper.

\section{Model Mismatch Analysis}

The mathematical model of PMSM [22] under the $d$ - $q$ axis of the rotating coordinate system is

$$
\begin{aligned}
& \left\{\begin{array}{l}
u_{d}=R_{0} i_{d}+\frac{\mathrm{d} \psi_{d}}{\mathrm{~d} t}-\omega \psi_{q}, \\
u_{q}=R_{0} i_{q}+\frac{\mathrm{d} \psi_{q}}{\mathrm{~d} t}+\omega \psi_{d},
\end{array}\right. \\
& \left\{\begin{array}{l}
\psi_{d}=L_{d} i_{d}+\psi_{f 0}, \\
\psi_{q}=L_{q} i_{q} .
\end{array}\right.
\end{aligned}
$$

Substitute formula (2) into formula (1) to get

$$
\left\{\begin{array}{l}
u_{d}=R_{0} i_{d}+L_{d} \frac{\mathrm{d} i_{d}}{\mathrm{~d} t}-\omega L_{q} i_{q} \\
u_{q}=R_{0} i_{q}+L_{q} \frac{\mathrm{d} i_{q}}{\mathrm{~d} t}+\omega L_{d} i_{d}+\omega \psi_{f 0} .
\end{array}\right.
$$

Among them, $u_{d}$ and $u_{q}$ are the stator $d$-q axis voltage components, $\Psi_{d}$ and $\Psi_{q}$ are the $d-q$ axis components of the stator flux linkage, $R_{0}$ is the stator resistance, $L_{d}$ and $L_{q}$ are inductances of $d-q$ axis, respectively. For a surface-mounted PMSM (SPMSM) control, the stator inductance meets $L_{d}=L_{q}=L_{0} . i_{d}$ and $i_{q}$ are, respectively, the stator $d$ - $q$ axis current components, $\Psi_{f 0}$ is the permanent magnet flux, $p$ is the number of pole pairs, $\omega$ is the motor speed, and $T_{e}$ is the electromagnetic torque.

Selecting the stator current as the state variable, according to formula (3), the following can be obtained:

$$
\left\{\begin{array}{l}
\dot{i_{d}}=\frac{u_{d}}{L_{0}}-\frac{R_{0} i_{d}}{L_{0}}+\frac{\omega L_{0} i_{q}}{L_{0}} \\
\dot{i_{q}}=\frac{u_{q}}{L_{0}}-\frac{R_{0} i_{q}}{L_{0}}+\omega i_{d}+\frac{\omega \psi_{f 0}}{L_{0}}, \\
\dot{i}=A i+B u+d,
\end{array}\right.
$$


where

$$
\begin{aligned}
& i=\left[\begin{array}{l}
i_{d} \\
i_{q}
\end{array}\right], \\
& u=\left[\begin{array}{l}
u_{d} \\
u_{q}
\end{array}\right] \text {, } \\
& d=\left[\begin{array}{c}
0 \\
\frac{-\omega \psi_{f 0}}{L}
\end{array}\right], \\
& A_{0}=\left[\begin{array}{cc}
\frac{-R_{0}}{L_{0}} & \omega \\
\omega & \frac{-R_{0}}{L_{0}}
\end{array}\right] \text {, } \\
& B_{0}=\left[\begin{array}{ll}
\frac{1}{L_{0}} & \omega \\
\omega & \frac{1}{L_{0}}
\end{array}\right] .
\end{aligned}
$$

The continuous time model (formula (5)) was discretized to obtain the state space model of the discrete time system:

$$
i(k+1)=A i(k)+B u(k)+T_{s} d(k),
$$

where

$$
\left\{\begin{array}{l}
A=e^{A_{0} T_{s}}=1+A_{0} T_{s}, \\
B=\int_{0}^{T_{s}} e^{A_{c} \tau} \mathrm{d} \tau \times B_{0} .
\end{array}\right.
$$

In the actual algorithm, the parameters used in the model are nominal values on the nameplate of the motor, and the predicted current is

$$
\left\{\begin{array}{l}
i_{d 0}(k+1)=\left(1-\frac{R_{0}}{L_{0}} T_{s}\right) i_{d}(k)+\frac{T_{s}}{L_{0}} u_{d 0}(k)+T_{s} \omega i_{q}(k), \\
i_{q 0}(k+1)=\left(1-\frac{R_{0}}{L_{0}} T_{s}\right) i_{q}(k)+\frac{T_{s}}{L_{0}} u_{q 0}(k)+\omega T_{s} i_{d}(k)-T_{s} \frac{\omega \psi_{f 0}}{L_{0}} .
\end{array}\right.
$$

The values of the parameters $R_{0}, L_{0}$, and $\Psi_{f 0}$ are the standard values on the motor nameplate.

However, in the actual control process, with the motor running, the parameters of the motor will change, and the predicted current is

$$
\left\{\begin{array}{l}
i_{d}(k+1)=\left(1-\frac{R}{L} T_{s}\right) i_{d}(k)+\frac{T_{s}}{L} u_{d}(k)+T_{s} \omega i_{q}(k), \\
i_{q}(k+1)=\left(1-\frac{R}{L} T_{s}\right) i_{q}(k)+\frac{T_{s}}{L} u_{q}(k)+\omega T_{s} i_{d}(k)-T_{s} \frac{\omega \psi_{f}}{L} .
\end{array}\right.
$$

The values of the parameters $R, L, \Psi_{f}$ are the actual values of the motor.

Due to the influence of temperature rise, flux saturation, and other factors, the nominal value on the nameplate of the motor will produce an error with the actual value. We make the control voltage equal and get the relationship between the actual predicted current and the ideal predicted current:

$$
\left\{\begin{array}{l}
u_{d}(k)=u_{d 0}(k), \\
u_{q}(k)=u_{q 0}(k) .
\end{array}\right.
$$

Then,

$$
\left\{\begin{array}{l}
i_{d}(k+1)=\frac{L_{0}}{L} i_{d 0}(k+1)+\frac{\Delta L-R T_{s}}{L} i_{d}(k)+\frac{\Delta L}{L} T_{s} \omega i_{q}(k), \\
i_{q}(k+1)=\frac{L_{0}}{L} i_{q 0}(k+1)+\frac{\Delta L-\Delta R T_{s}}{L} i_{q}(k)-\frac{\Delta L}{L} T_{s} \omega i_{d}(k)-\frac{\Delta \psi_{f}}{L} T_{s} \omega,
\end{array}\right.
$$

where $\Delta L=L-L_{0}, \Delta R=R-R_{0}$, and $\Delta \Psi_{f}=\Psi_{f}-\Psi_{f 0}$. Because the sampling time $\mathrm{T}_{\mathrm{s}}$ is small enough and $i_{d}=0$, the $\mathrm{Z}$ transform of equation (12) can be used to obtain the following equation:

$$
\left\{\begin{array}{l}
z \times i_{d}(z)=z \frac{L_{0}}{L} i_{d 0}(z)+\frac{\Delta L}{L} i_{d}(z), \\
z \times i_{q}(k+1)=z \frac{L_{0}}{L} i_{q 0}(z)+\frac{\Delta L}{L} i_{q}(k) .
\end{array}\right.
$$

Therefore, the transfer function between the actual value of current prediction and the nominal value is obtained as

$$
\frac{i_{d, q}(z)}{i_{d 0, q 0}(z)}=\frac{\left(L_{0} / L\right) \times z}{z+\left(\left(L_{0} / L\right)-1\right)} .
$$

To maintain the stability of the discrete system, the poles of the discrete system are located in the unit circle, that is, $\left|\left(L_{0} / L\right)-1\right| \leq 1$; when $0 \leq L_{0} \leq 2 L$, the system is in a stable state, and when the inductance value on the nameplate of the motor is 
greater than twice the actual inductance value, the system will be unstable, resulting in oscillation and current static difference.

\section{Mathematical Model of PMSM Based on Parameter Disturbance}

When parameter perturbation is considered, the PMSM mathematical model changes from formula (1) to

$$
\left\{\begin{array}{l}
u_{d}=\left(R_{0}+\Delta R\right) i_{d}+\left(L_{0}+\Delta L\right) \frac{\mathrm{d} i_{d}}{\mathrm{~d} t}-\omega\left(L_{0}+\Delta L\right) i_{q}, \\
u_{q}=\left(R_{0}+\Delta R\right) i_{q}+\left(L_{0}+\Delta L\right) \frac{\mathrm{d} i_{q}}{\mathrm{~d} t}+\omega\left(L_{0}+\Delta L\right) i_{d}+\omega\left(\psi_{f 0}+\Delta \psi_{f}\right),
\end{array}\right.
$$

where $R_{0}, L_{0}$, and $\Psi_{f 0}$, respectively, correspond to the motor nameplate resistance, inductance, and magnetic flux calibration values and $\Delta R, \Delta L$, and $\Delta \Psi_{f}$ are parameter change values. Formula (15) simplifies to

$$
\left\{\begin{array}{l}
\frac{\mathrm{d} i_{d}}{\mathrm{~d} t}=\frac{u_{d}}{L_{0}}+\left(-\frac{\Delta L}{L_{0}} \frac{\mathrm{d} i_{d}}{\mathrm{~d} t}-\frac{\left(R_{0}+\Delta R\right) i_{d}}{L_{0}}+\frac{\omega\left(L_{0}+\Delta L\right) i_{q}}{L_{0}}\right) \\
\frac{\mathrm{d} i_{q}}{\mathrm{~d} t}=\frac{u_{q}}{L_{0}}+\left(-\frac{\Delta L}{L_{0}} \frac{\mathrm{d} i_{q}}{\mathrm{~d} t}-\frac{\left(R_{0}+\Delta R\right) i_{q}}{L_{0}}+\frac{\omega\left(L_{0}+\Delta L\right) i_{d}}{L_{0}}+\frac{\omega\left(\psi_{f 0}+\Delta \psi_{f}\right)}{L_{0}}\right)
\end{array}\right.
$$

where the $d-q$ axis current is the state variable and the $d-q$ axis voltage is the control variable. If the remaining parameter variation terms, which correspond to the total disturbance of parameter changes, are expressed as $E_{d}$ and $E_{q}$, formula (16) can be simplified into

$$
\left\{\begin{array}{l}
\frac{\mathrm{d} i_{d}}{\mathrm{~d} t}=\frac{u_{d}}{L_{0}}+E_{d} \\
\frac{\mathrm{d} i_{q}}{\mathrm{~d} t}=\frac{u_{q}}{L_{0}}+E_{q}
\end{array}\right.
$$

where

$$
\left\{\begin{array}{l}
E_{d}=-\frac{\Delta L}{L_{0}} \frac{\mathrm{d} i_{d}}{\mathrm{~d} t}-\frac{\left(R_{0}+\Delta R\right) i_{d}}{L_{0}}+\frac{\omega\left(L_{0}+\Delta L\right) i_{q}}{L_{0}}, \\
E_{q}=-\frac{\Delta L}{L_{0}} \frac{\mathrm{d} i_{q}}{\mathrm{~d} t}-\frac{\left(R_{0}+\Delta R\right) i_{q}}{L_{0}}+\frac{\omega\left(L_{0}+\Delta L\right) i_{d}}{L_{0}}+\frac{\omega\left(\psi_{f 0}+\Delta \psi_{f}\right)}{L_{0}} .
\end{array}\right.
$$

Taking the $d$-axis as an example, an extended state observer is established [23-25], and its state space equation is

$$
\left\{\begin{array}{l}
\Delta m_{d}=z_{1 d}-i_{d} \\
\dot{z_{1 d}}=z_{2 d}+b u_{d}-\beta_{1 d} \Delta m_{d} \\
\dot{z_{2 d}}=-\beta_{2 d} \Delta m_{d}
\end{array}\right.
$$

where $z_{1 d}$ is the observed value of $i_{d}, z_{2 d}$ is the observed value of $E_{d}$, and $\beta_{1 d}$ and $\beta_{2 d}$ are the gains of each state observer, respectively.
To enhance the antidisturbance ability of the system and neutralize the interference caused by the uncertain factors of the system, a nonlinear error feedback control rate is designed:

$$
\left\{\begin{array}{l}
u_{0}=\beta_{3 d}\left(i_{d}^{*}-i_{d}\right) \\
u_{d}=\frac{u_{0}-z_{2 d}}{b}
\end{array}\right.
$$

where $\beta_{3 d}$ represents the system's unknown interference gain.

Consistent with the discretization method of formula (5), discretization is carried out in formula (19) to obtain the discretization state space equation as

$$
\left\{\begin{array}{l}
\Delta m_{d}(k)=z_{1 d}(k)-i_{d}(k), \\
z_{1 d}(k+1)=z_{1 d}(k)+T_{s} \times\left\{z_{2 d}(k)+b u_{d}(k)-\beta_{1 d} \Delta m_{d}(k)\right\}, \\
z_{2 d}(k+1)=z_{2 d}(k)-T_{s} \beta_{2 d} \Delta m_{d}(k) .
\end{array}\right.
$$

Applying the $Z$ transformation to formula (21) yields

$$
\left\{\begin{array}{l}
\Delta m_{d}(z)=z_{1 d}(z)-i_{d}(z) \\
z \cdot z_{1 d}(z)=z_{1 d}(z)+T_{s} \times\left\{z_{2 d}(z)+b u_{d}(z)-\beta_{1 d} \Delta m_{d}(z)\right\} \\
z \cdot z_{2 d}(z)=z_{2 d}(z)-T_{s} \beta_{2 d} \Delta m_{d}(z)
\end{array}\right.
$$

Then, the transfer function of the discrete system is

$$
G_{1}(z)=\frac{z_{1 d}(z)}{i_{d}(z)}=\frac{\beta_{1} z+\beta_{2} T_{s}-\beta_{1}}{(z-1)^{2}+\beta_{1} z+\beta_{2} T_{s}-\beta_{1}} .
$$

where $\beta_{1}=\beta_{1 d} T_{s}$ and $\beta_{2}=\beta_{2 d} T_{s}$. 
To maintain the stability of the discrete system, the poles of the discrete system should be located in the unit circle, and hence the characteristic equation can be obtained as

$$
(z-1)^{2}+\beta_{1} z+\beta_{2} T_{s}-\beta_{1}=0 .
$$

To apply the Routh-Hurwitz criterion, let $z=((\lambda+1) /(\lambda-1))$, thus yielding the characteristic equation of $\lambda$ domain as

$$
\beta_{2} T_{s} \lambda^{2}+2\left(\beta_{1}-\beta_{2} T_{s}\right) \lambda+\left(4+\beta_{2} T_{s}-\beta_{1}\right)=0
$$

According to the Rolls-Helvitz criterion, each coefficient of the order equation is greater than 0 , and the range of gain coefficients is obtained by

$$
\left\{\begin{array}{l}
\beta_{2} T_{s}<\beta_{1}<4+\beta_{2} T_{s} \\
\beta_{2}>0
\end{array}\right.
$$

The total perturbation observations $z_{2 d}$ and $z_{2 q}$ of parameter changes are substituted into formula (17) and discretized to obtain the predicted current value based on parameter perturbations, that is,

$$
\left\{\begin{array}{l}
i_{d}(k+1)=i_{d}(k)+\frac{T_{s}}{L_{0}} u_{d}(k)+T_{s} z_{2 d}, \\
i_{q}(k+1)=i_{q}(k)+\frac{T_{s}}{L_{0}} u_{q}(k)+T_{s} z_{2 q} .
\end{array}\right.
$$

Then, according to formula (27), the required $d$ - $q$ control voltage is obtained as

$$
\left\{\begin{array}{l}
u_{d}(k)=\frac{L_{0}}{T_{s}}\left\{i_{d}(k+1)-i_{d}(k)\right\}-L_{0} z_{2 d}, \\
u_{q}(k)=\frac{L_{0}}{T_{s}}\left\{i_{q}(k+1)-i_{q}(k)\right\}-L_{0} z_{2 q} .
\end{array}\right.
$$

When $i_{d}(k+1)=i_{d}^{*}$ and $i_{q}(k+1)=i_{q}^{*}$, where $i_{d}^{*}$ and $i_{q}^{*}$ are the output currents of the PMSM speed ring, for the control needed when the current values $i_{d}^{*}$ and $i_{q}^{*}$ are output, the voltage is obtained by

$$
\left\{\begin{array}{l}
u_{d}^{*}=\frac{L_{0}}{T_{s}}\left\{i_{d}^{*}-i_{d}(k)\right\}-L_{0} z_{2 d} \\
u_{q}^{*}=\frac{L_{0}}{T_{s}}\left\{i_{q}^{*}-i_{q}(k)\right\}-L_{0} z_{2 q} .
\end{array}\right.
$$

The optimal basic voltage is finally obtained.

The MPC based on parametric disturbance compensation is shown in Figure 1.

\section{Control Delay Compensation}

In the process of digital system control, there is a beat delay between the actual output instruction and the control instruction; therefore, the model prediction method was used to compensate this delay [26]. The specific method is given as follows: firstly, $u_{d}(k)$ and $u_{q}(k)$ were used to calculate the prediction current $i_{d}(k+1)$ and $i_{q}(k+1)$ at the next moment of the $d$ - $q$ axis. Using (29), $i_{d}(k)$ and $i_{q}(k)$ were replaced in $i_{d}$ $(k+1)$ and $i_{q}(k+1)$, respectively, and $u_{d}^{*}$ and $u_{q}^{*}$ were obtained. The algorithm flow of the proposed method is shown in Figure 2.

\section{Analysis of PMSM Model Based on the Frequency-Domain Method}

5.1. Convergence Analysis of PMSM Model. According to formulas (17) and (19),

$$
\Delta \dot{m}_{d}=\dot{z_{1 d}}-\dot{i_{d}}=z_{2 d}-\beta_{1 d} \Delta m_{d}-E_{d}
$$

Consistent with the discretization method of formula (5), the discretized state space equation of formula (30) is

$$
\Delta m_{d}(k+1)=\left(1-\beta_{1 d} T_{s}\right) \Delta m_{d}(k)+T_{s}\left(z_{2 d}-E_{d}\right) .
$$

Formula (31) is transformed by $Z$ :

$$
z \cdot \Delta m_{d}(z)=\left(1-\beta_{1 d} T_{s}\right) \Delta m_{d}(z)+T_{s}\left\{z_{2 d}(z)-E_{d}(z)\right\} .
$$

Combined with formula (22), the closed-loop transfer function can be obtained as

$$
G_{2}(z)=\frac{z_{2 d}(z)}{E_{d}(z)}=\frac{\beta_{2} T_{s}}{(z-1)^{2}+\beta_{1} z+\beta_{2} T_{s}-\beta_{1}}
$$

Substituting $z=j \omega$ into formulas (23) and (33) yields

$$
\left\{\begin{array}{l}
G_{1}(j \omega)=\frac{z_{1 d}(j \omega)}{i_{d}(j \omega)}=\frac{j \beta_{1} \omega+\beta_{2} T_{s}-\beta_{1}}{(j \omega-1)^{2}+j \beta_{1} \omega+\beta_{2} T_{s}-\beta_{1}}=\frac{(j \omega-1) \beta_{1}}{\left(1-\omega^{2}-2 j \omega\right)+\beta_{2} T_{s}+(j \omega-1) \beta_{1}}, \\
G_{2}(j \omega)=\frac{z_{2 d}(j \omega)}{E_{d}(j \omega)}=\frac{\beta_{2} T_{s}}{(j \omega-1)^{2}+j \beta_{1} \omega+\beta_{2} T_{s}-\beta_{1}}=\frac{\beta_{2} T_{s}}{\left(1-\omega^{2}-2 j \omega\right)+\beta_{2} T_{s}+(j \omega-1) \beta_{1}} .
\end{array}\right.
$$




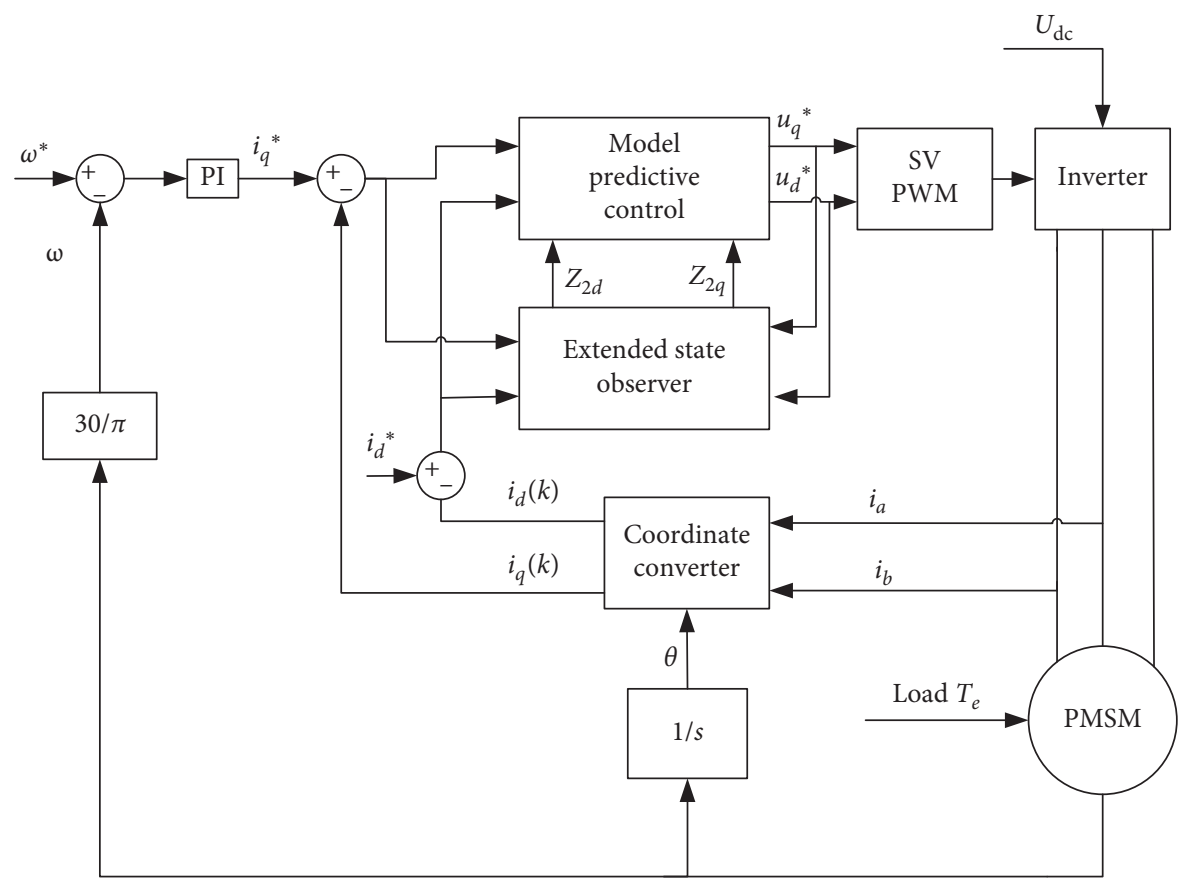

FIGURE 1: MPC based on parametric disturbance compensation.

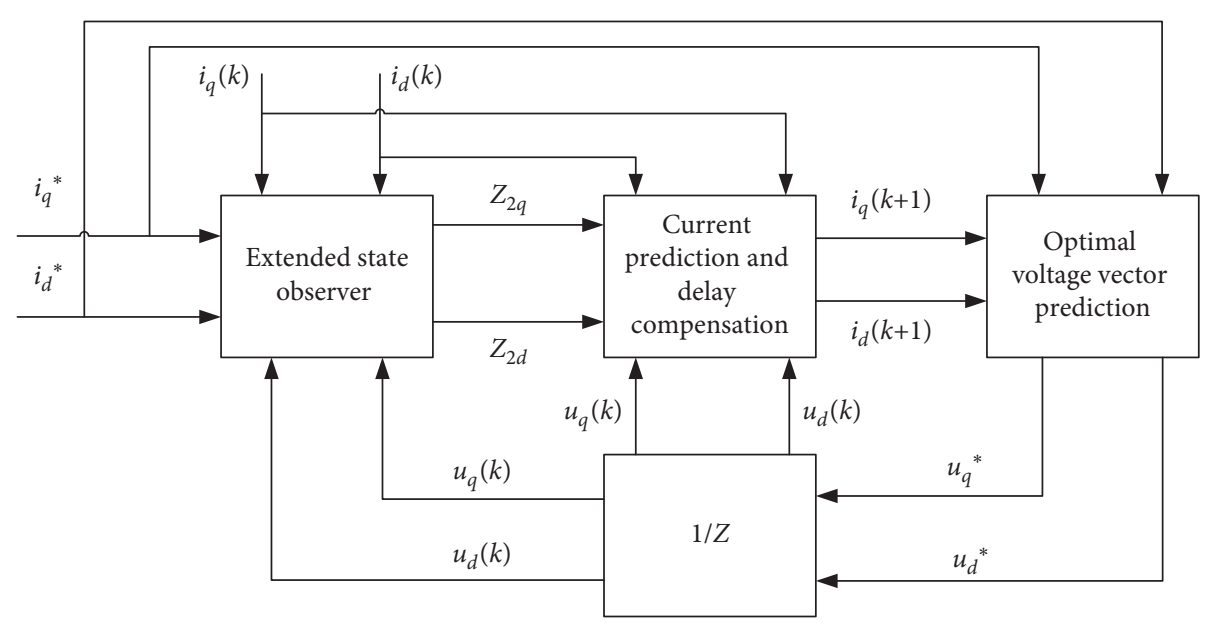

FIgURE 2: Flowchart of control delay compensation method.

According to the knowledge of classical control theory, when the maximum cutoff frequency of $\omega_{\max }$ at the $i$-th channel in the control system and the gain parameters $\beta_{1}$ and $\beta_{2}$ satisfy $\min \left\{\beta_{1}, \beta_{2}\right\} \gg \omega_{\max }$, then under the condition $\omega \leq \omega_{\max }$, the system transfer function is

$$
\left\{\begin{array}{l}
G_{1}(j \omega) \approx 1, \\
G_{2}(j \omega) \approx 1 .
\end{array}\right.
$$

It can be observed from formula (35) that the control system converges and is stable at this time, $z_{1 d} \longrightarrow i_{d}, z_{2 d} \longrightarrow E_{d}$. This shows that the observed values obtained by the constructed system model reflect the true values more accurately.

In the PMSM motion control system, the speed control loop is used as the outer loop and the current control loop is used as the inner loop. The purpose of the speed control loop is to control the speed of the motor so that it can both regulate and stabilize the speed. The purpose of the current control loop is to focus on the dynamic adjustment process of the control system so that the stator current can track the given current vector more accurately and quickly. To achieve a good control effect, the current inner loop control cycle should generally be short, the bandwidth should be high, and the speed outer loop bandwidth should be low. The common PMSM has a current loop bandwidth of $2.5 \mathrm{kHz}$ and a speed loop bandwidth of $400 \mathrm{~Hz}$. According to the analysis of the actual situation, the proposed model is feasible and meets the convergence requirement.

\subsection{Filtering Performance Analysis of PMSM Model.} During the operation of the motor, the parameter drift and the disturbance of external noise will cause the model 
mismatch of the motor model established above, hence the need to analyze the filterability and robustness of the established PMSM model. According to formula (19),

$$
\left\{\begin{array}{l}
z_{1 d} \cdot s=-s^{-1} \beta_{2 d} \cdot z_{1 d}+s^{-1} \beta_{2 d} \cdot i_{d}+b u_{d}-\beta_{1 d} \cdot z_{1 d}+\beta_{1 d} \cdot i_{d}, \\
z_{2 d} \cdot s=-\beta_{2 d} \cdot z_{1 d}+\beta_{2 d} \cdot i_{d} .
\end{array}\right.
$$

This can be further simplified to

$$
\left\{\begin{array}{l}
z_{1 d}=\frac{\beta_{1 d} \cdot s+\beta_{2 d}}{s^{2}+\beta_{1 d} \cdot s+\beta_{2 d}} i_{d}+\frac{b s}{s^{2}+\beta_{1 d} \cdot s+\beta_{2 d}} u_{d} \\
z_{2 d}=\frac{\beta_{2 d} \cdot s}{s^{2}+\beta_{1 d} \cdot s+\beta_{2 d}} i_{d}-\frac{b \beta_{2 d}}{s^{2}+\beta_{1 d} \cdot s+\beta_{2 d}} u_{d}
\end{array}\right.
$$

It can be seen from formula (37) that the state observers $z_{1 d}$ and $z_{2 d}$ are related to the measured value $i_{d}$ and the input value $u_{d}$. Because this paper focuses on the analysis of model mismatch caused by parameter disturbance, the filter and robustness of the parameter disturbance observer will be analyzed next.

Characterize the transfer function of the state observer $z_{2 d}$ as

$$
\left\{\begin{array}{l}
f_{2 d 1}=\frac{\beta_{2 d} \cdot s}{s^{2}+\beta_{1 d} \cdot s+\beta_{2 d}} \\
f_{2 d 2}=\frac{b \beta_{2 d}}{s^{2}+\beta_{1 d} \cdot s+\beta_{2 d}}
\end{array}\right.
$$

The characteristic equations of $f_{2 d 1}$ and $f_{2 d 2}$ transfer functions are

$$
s^{2}+\beta_{1 d} \cdot s+\beta_{2 d}=0
$$

According to the classical control theory, the speed of convergence of the observer for $z_{2 d}$ estimation is directly related to the characteristic equation. Define the damping ratio $\xi$ as

$$
\xi=\frac{\beta_{1 d}}{2 \sqrt{\beta_{2 d}}} .
$$

The damping ratio $\xi$ is divided into three states: underdamping, critical damping, and overdamping. The transfer function of the observer $z_{2 d}$ operates in these three states, as shown in the amplitude-frequency characteristics and phase-frequency characteristics of its Bode diagram. By fixing $\beta_{1 d}=10, \xi$ becomes equal to $0.707,1$, and 1.770 , corresponding to the three states of the damping ratio. At this time, using formula (40) can yield $\beta_{2 d}=50.015,25$, and 7.980 .

The Bode diagram of the measurement error transfer function $f_{2 d 1}$ due to the measurement value $i_{d}$ is shown in Figure 3.

Because the noise signal is generally a high-frequency signal, the amplitude of the logarithmic amplitude frequency of the system transfer function in the high-frequency band directly reflects the suppression effect of the system on the high-frequency noise signal. Lower amplitudes correspond to an increased ability of the system to suppress higherfrequency noise signals. The characteristics near the cutoff frequency determine the stability of the dynamic response of the system, and the size of the cutoff frequency determines the fast response. With the decrease in the $\beta_{2 d}$ value, the system operates under the three states: underdamping, critical damping, and overdamping. These three states change little in the low-frequency band. In contrast, the amplitude gradually decreases in the high-frequency band, which means that the attenuation characteristics increase, thus increasing its ability to suppress high-frequency noise. However, the cutoff frequency of the system decreases in turn, so the rapidity of the system's dynamic response gradually decreases. In the overdamped state, the system's ability to suppress high-frequency noise is enhanced, and the anti-interference ability is enhanced as well; however, the dynamic response characteristics are slower, and the stability is poor, while in the underdamped state, the system's antiinterference ability and robustness are reduced, and the dynamic response is faster. Therefore, according to the research needs, appropriate parameters can be debugged to obtain the desired anti-interference ability and dynamic response speed.

Input perturbations caused by the input value $u_{d}$. By observing the molecules of its characteristic function $f_{2 d 2}$, the transition frequency of the transfer function $f_{2 d 2}$, namely, $\sqrt{b \beta_{2 d}}$, will not change and the low-frequency and highfrequency characteristics of the system are directly related to this transition frequency. Therefore, the low-frequency and high-frequency characteristics of the system are unchanged. However, when $\beta_{1 d}$ is fixed and different $\beta_{2 d}$ values are selected, different system characteristics will be obtained. It can be seen from Figure 4 that with the decrease of $\beta_{2 d}$, the system operates in the three states: underdamping, critical damping, and overdamping in turn. The system is in a lowfrequency band, and its characteristics are unchanged, similar to a low-pass filter. Compared with the underdamped state, the overdamped state has a higher amplitude in the high-frequency range, but the cutoff frequency is larger; therefore, the system has a stronger anti-interference ability in the overdamped state.

5.3. Selection of Gain Parameters. According to the above analysis, the anti-interference ability of the system and the its dynamic response speed are not only related to $\beta_{1 d}$ and $\beta_{2 d}$ parameter values but also to the relationship between them. When the system's damping ratio is in different states, different system characteristics will be obtained. The bandwidth of the observer and the bandwidth of the controller can be used to set the parameters. When the natural frequency $\omega_{0}$ of the system channel is known and the system satisfies formula (35), the system converges. To improve the stability of the system, $\beta_{2 d}=\omega_{0}^{2}$ is selected. Taking the underdamped system as an example, let $\xi=0.707$, we can obtain $\beta_{1 d}$ from equation (40). Under this condition, the system is underdamped, and the dynamic response speed is fast, but the anti-interference 

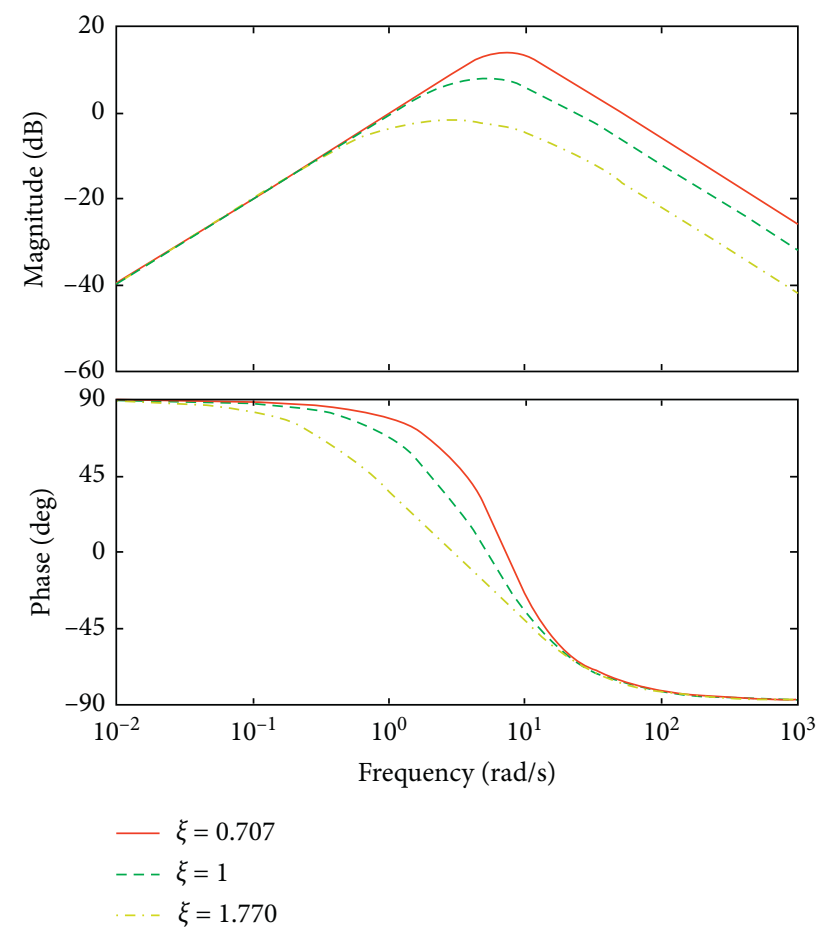

FIgURE 3: The noise frequency characteristics measured under different $\xi$ conditions.

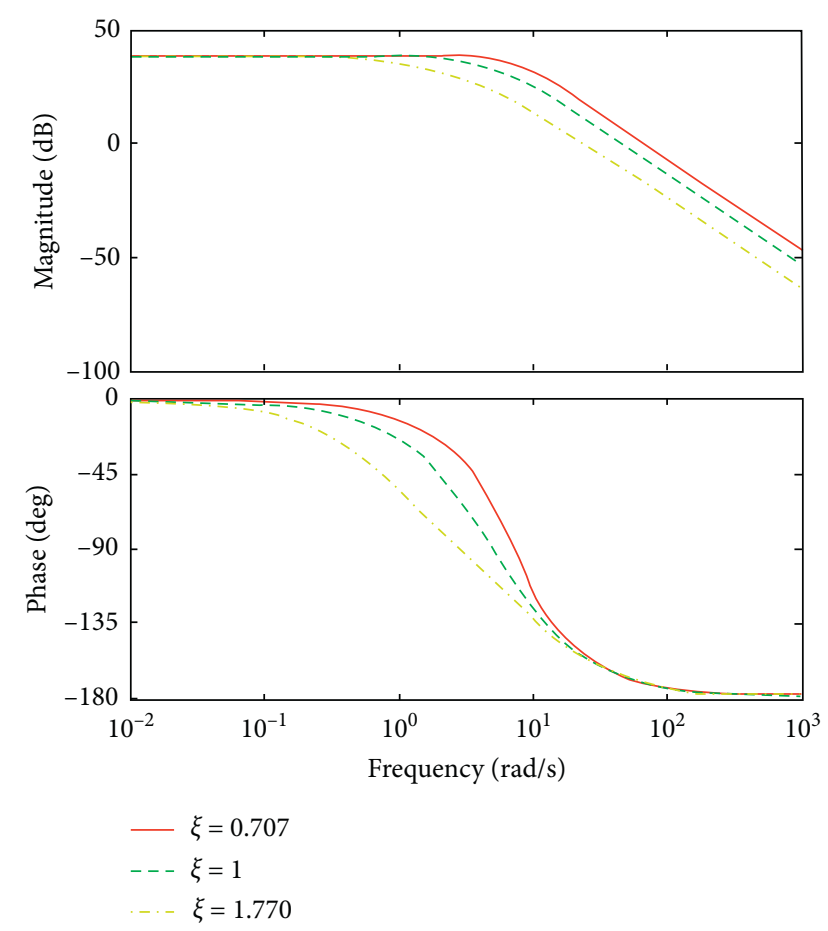

FIGURE 4: Input disturbance frequency characteristics under different $\xi$ conditions.

ability and robustness are poor. Therefore, according to the requirements, the amplitude-frequency characteristics and phase-frequency characteristics should be analyzed before changing the parameters to achieve a balanced compromise.

\section{Simulation Verification}

To verify the effectiveness of the method proposed in this paper, MATLAB/Simulink simulation software was used for the simulation experiment. The simulation experiment parameters are listed in Table 1 . The inductance disturbance was set as a straight slope from $0.5 L_{0}$ to $2.5 L_{0}$ during a time interval from $0.1 \mathrm{~s}$ to $0.3 \mathrm{~s}$.

First, only continuous inductance disturbance was considered in a no-load scenario. As can be seen from Figure 5, due to the influence of the inductance disturbance, the traditional vector control method showed a large ripple in the motor speed, electromagnetic torque, and $d-q$ axis current, which is not conducive to the stable control of the motor. The method presented in this paper shows good tracking, smoothness, and stability of the running curve. As shown in Figure 5(a), the rated speed set by the motor was $1000 \mathrm{rad} / \mathrm{s}$. During the period of inductance disturbance, the method proposed in this paper maintained stable rated speed and the motor ran smoothly.

The method presented in this paper has better dynamic characteristics than the vector control method in a no-load condition. To better verify the method proposed in this paper, under the condition of continuous inductance disturbance, load torque was added.

According to the simulation results, as shown in Figure 6(a), under the continuous inductance disturbance, the dynamic curve proposed in this paper has better tracking, stability, and smaller pulsation than the vector control method. When load torque was added at $t=0.2 \mathrm{~s}$, although both methods show a decrease in speed, the method proposed in this paper has a small decrease and a 
TABLE 1: Simulation parameters.

\begin{tabular}{lcc}
\hline Symbol & Quantity & Numerical value \\
\hline$T_{s}$ & Sampling period & $100 \mu \mathrm{s}$ \\
$p$ & Number of pole pairs & 4 \\
$R_{0}$ & Stator resistance & $0.958 \Omega$ \\
$\Psi_{f 0}$ & Permanent magnet flux & $0.1827 \mathrm{~Wb}$ \\
$L_{0}$ & Electrical inductance & $12 \mathrm{mH}$ \\
$J$ & Rotational inertia & $0.003 \mathrm{~kg} \cdot \mathrm{m}^{2}$ \\
$U_{\mathrm{dc}}$ & DC bus voltage & $311 \mathrm{~V}$ \\
$\Omega$ & Motor speed & $1000 \mathrm{rad} / \mathrm{s}$ \\
\hline
\end{tabular}

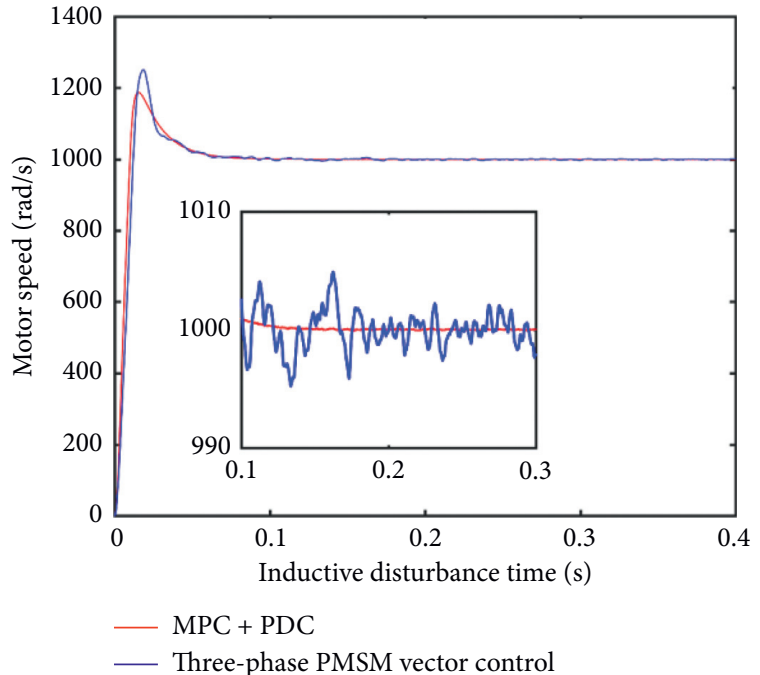

(a)

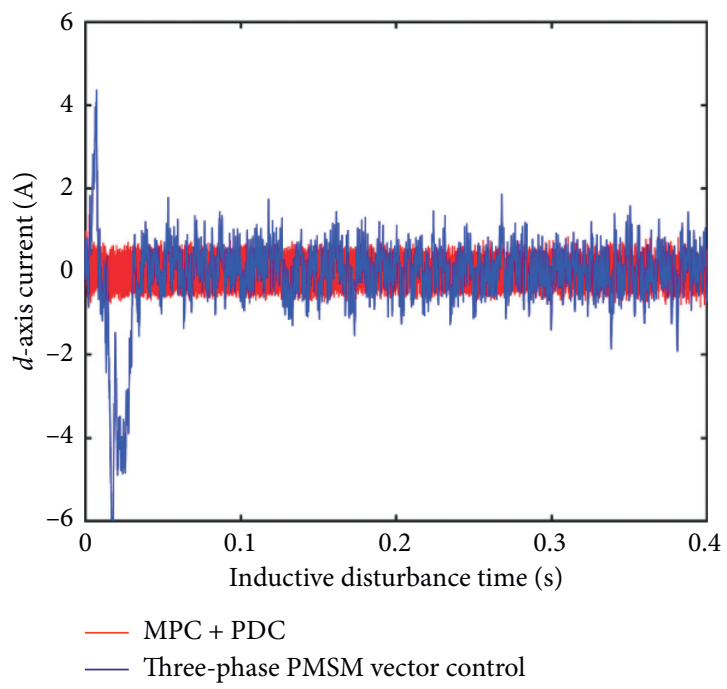

(c)

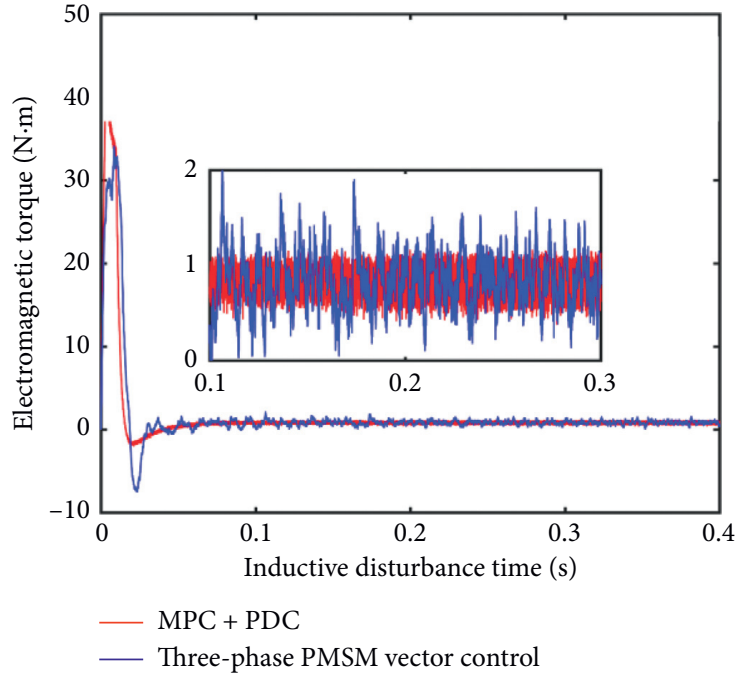

(b)

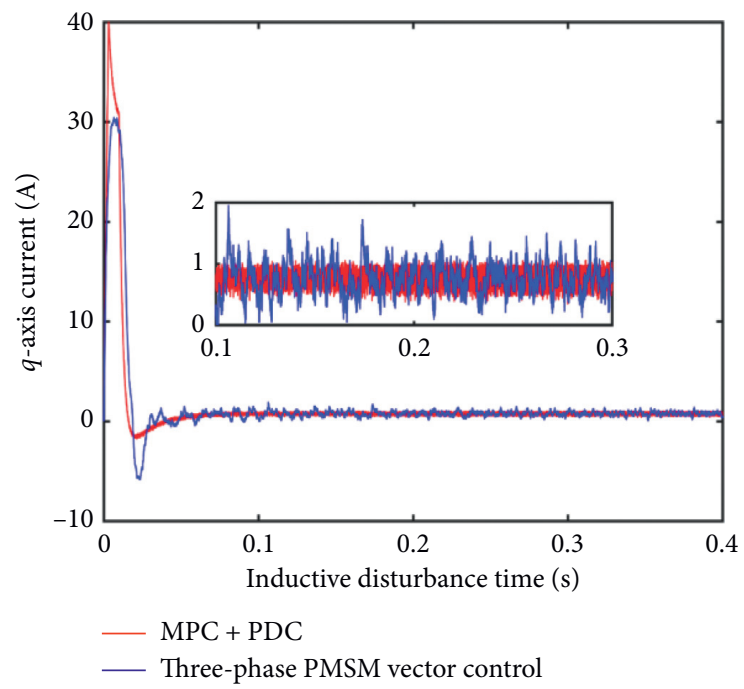

(d)

Figure 5: The motor response waveforms of the two proposed methods: (a) motor speed; (b) electromagnetic torque; (c) $d$-axis current; (d) q-axis current.

fast dynamic response, which allows the system to recover the rated speed quickly, indicating that the proposed method has a good dynamic performance and anti-interference ability. In Figure 6(b): electromagnetic torque dynamic curve, the method proposed in this paper shows good stability. It follows the load torque after approximately $0.02 \mathrm{~s}$ and has a fast response speed. However, in both methods, the load torque of $T_{L}=10 \mathrm{~N} \cdot \mathrm{m}$ shows a slight overshoot. In Figure 6(c): measurement of $d$-axis current, the method proposed in this paper shows a small ripple and relatively 


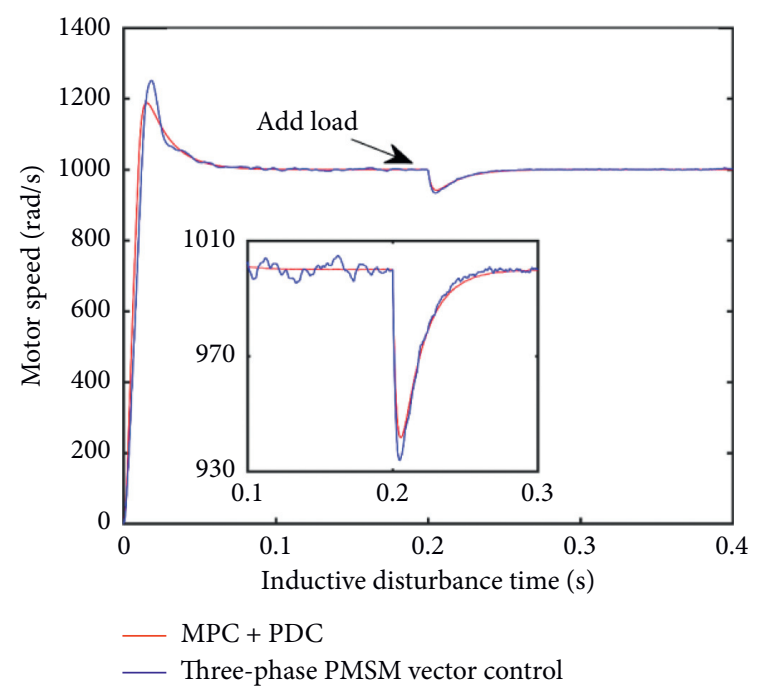

(a)

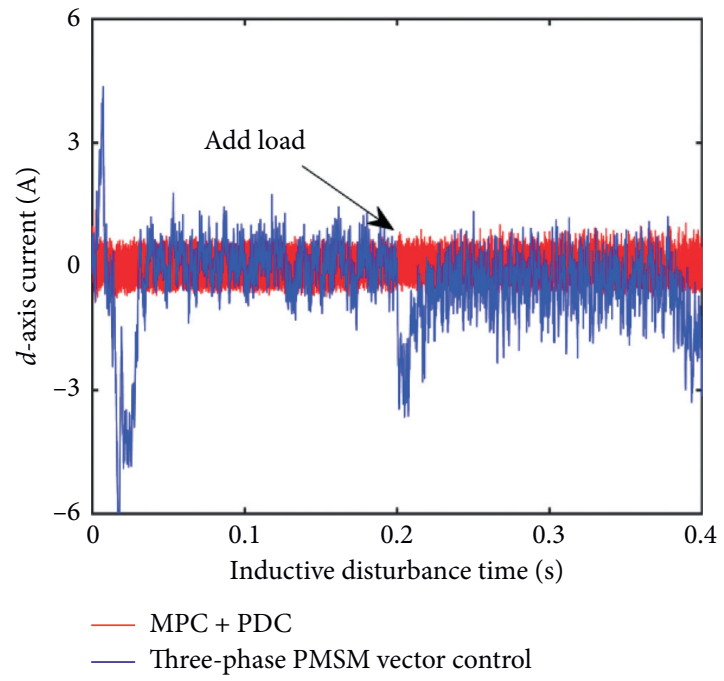

(c)

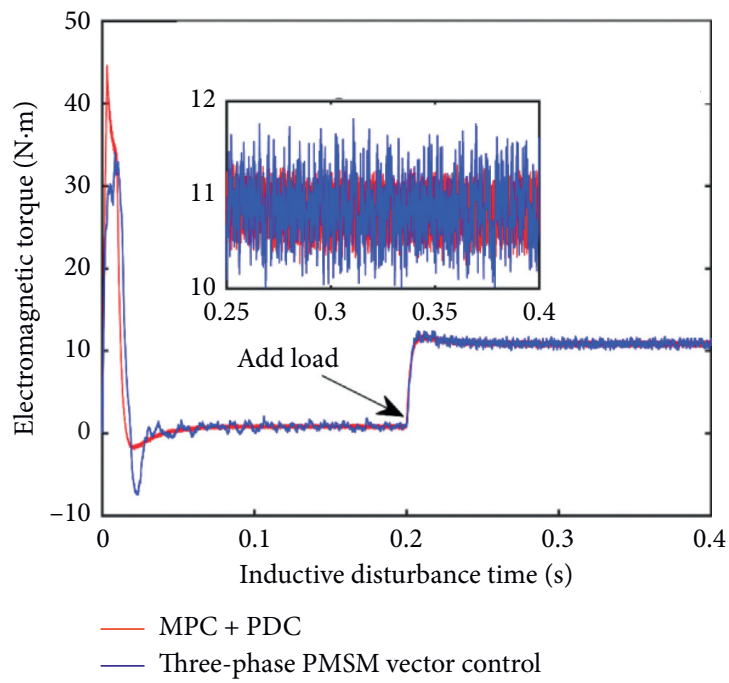

(b)

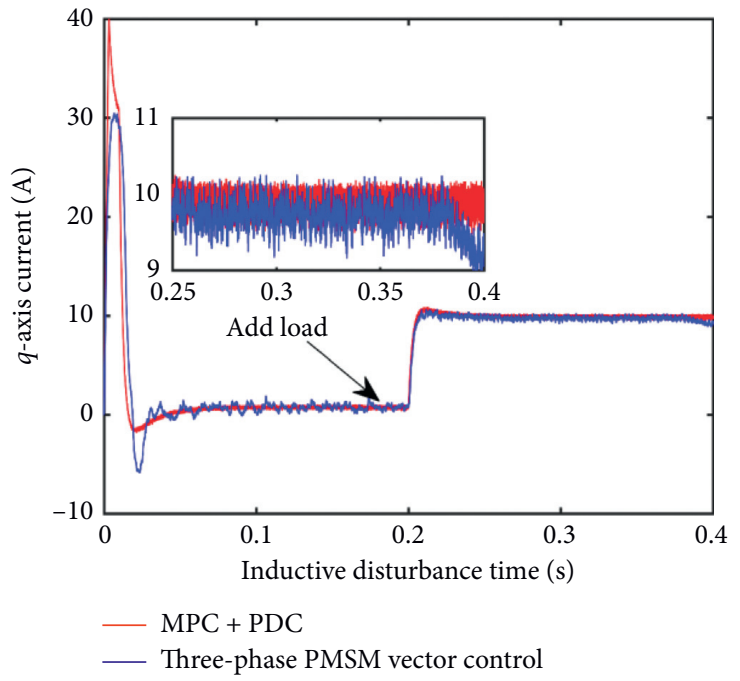

(d)

Figure 6: The motor response waveform of the two methods when load is added: (a) motor speed; (b) electromagnetic torque; (c) $d$-axis current; (d) $q$-axis current.

stable current following the rated $d$-axis. When $t=0.2 \mathrm{~s}$ and load torque is added, the method presented in this paper shows better stability, can maintain the rated current, and has better anti-interference ability and dynamic performance. However, the traditional vector control method has a large imbalance and takes a long time to return to the rated current. As for Figure 6(d), the method proposed in this paper has better overall stability; after adding load, the method proposed in this paper has a small overshoot and can quickly recover to the rated state.

The drift of the parameter inductance and the magnet flux linkage leads to the model mismatch, which has a greater impact on the control performance of the motor, while the mismatch of the stator resistance has a small impact on the control performance of the motor, which is almost negligible [27]. Figure 7 shows the robustness and anti-interference ability of the method proposed in this paper in the case of magnet flux linkage mismatch and external load disturbance. The magnet flux linkage disturbance was set as a straight slope from $0.5 \psi_{f 0}$ to $2.5 \psi_{f 0}$ during a time interval from $0.1 \mathrm{~s}$ to $0.3 \mathrm{~s}$. In Figure 7(a), under the continuous disturbance of the magnet flux linkage, the vector control shows large speed pulsation, while the MPC + PDC method has a smooth operating curve without large pulsation. In Figure $7(b)$, the vector control method produces larger torque ripple, while the MPC + PDC method has less torque ripple. When external load disturbances are added, it can quickly track and maintain smooth operation. In Figures 7(c) and 7(d), the vector control 


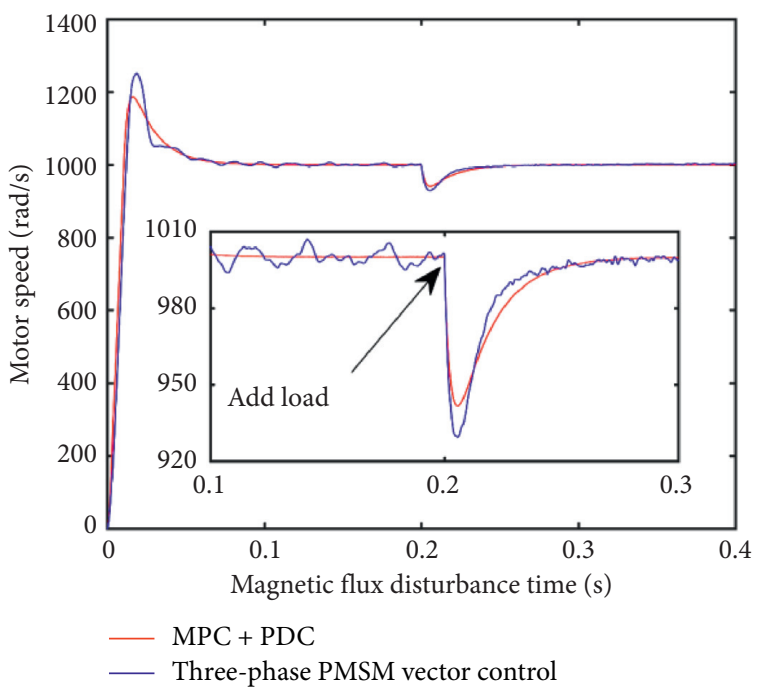

(a)

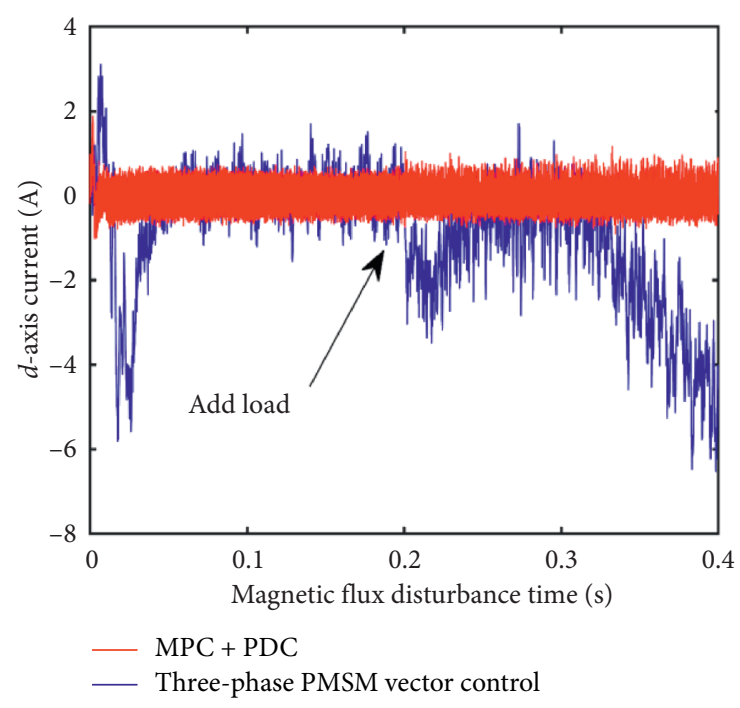

(c)

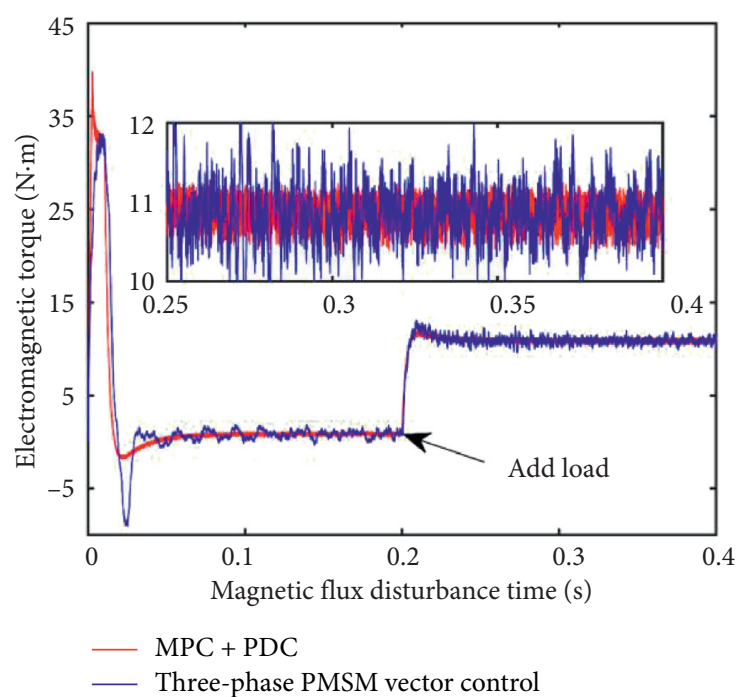

(b)

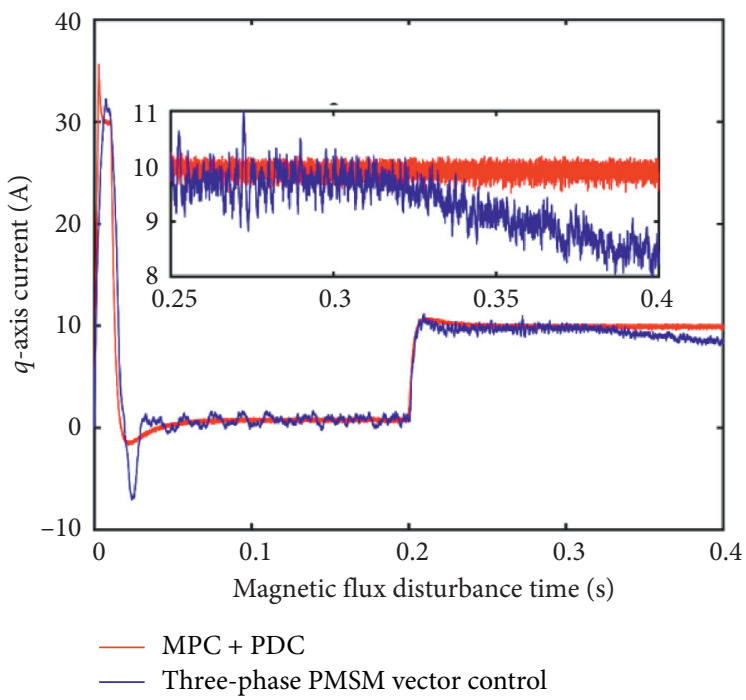

(d)

Figure 7: The motor response waveform of the two methods when load is added: (a) motor speed; (b) electromagnetic torque; (c) $d$-axis current; (d) $q$-axis current.

method produces a larger current ripple, and the current tracking effect is poor. The $d-q$ axis current cannot accurately track the rated current under the disturbance of the magnet flux linkage and external load disturbance, and the stability is poor. However, the MPC + PDC method does not produce large current ripples under interference, maintains a good tracking effect, and has strong robustness and anti-interference ability.

Figure 8 shows the comparison between the MPC method and the MPC + PDC method proposed in this paper under the condition of model mismatch and external load disturbance. In the motor starting phase, the MPC method produces a large overshoot, which is not conducive to the smooth operation of the motor. In the working phase, he MPC method produces large torque ripples and current ripples, with low robustness and anti-interference ability, while MPC + PDC runs relatively smoothly, with better tracking effect, strong robustness, and anti-interference ability.

The simulation results show that MPC based on parametric disturbance compensation has good stability and followability under the interference of continuous inductance and can maintain good performance when adding load, showing good anti-interference ability and fast dynamic response. 


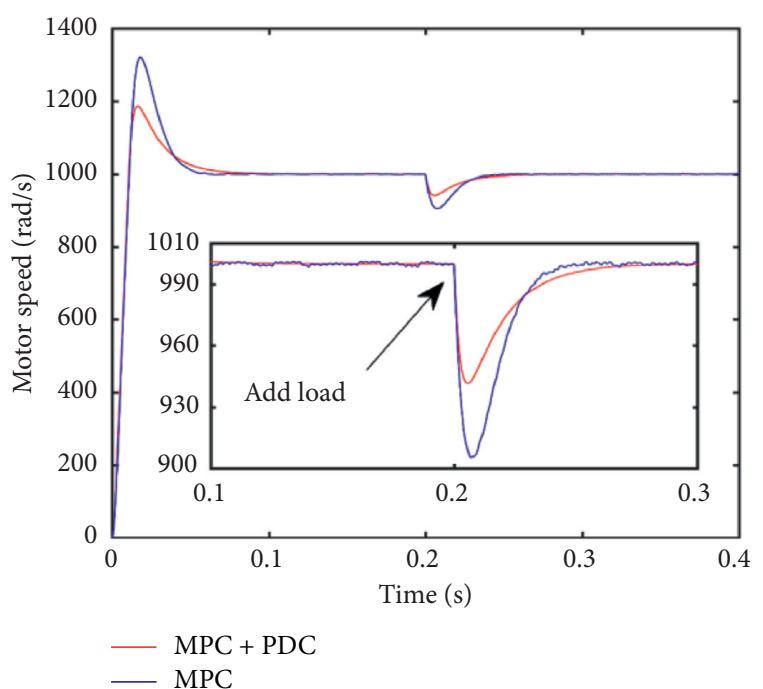

(a)

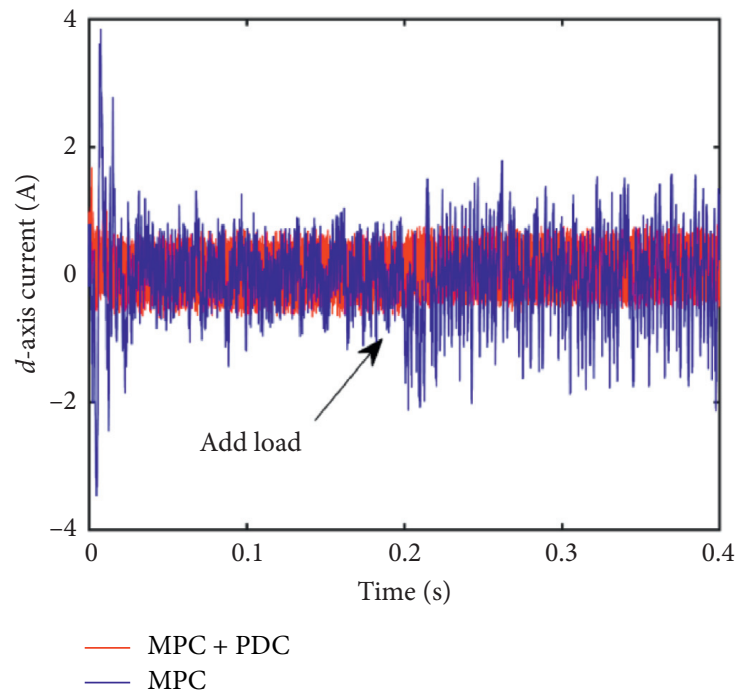

(c)

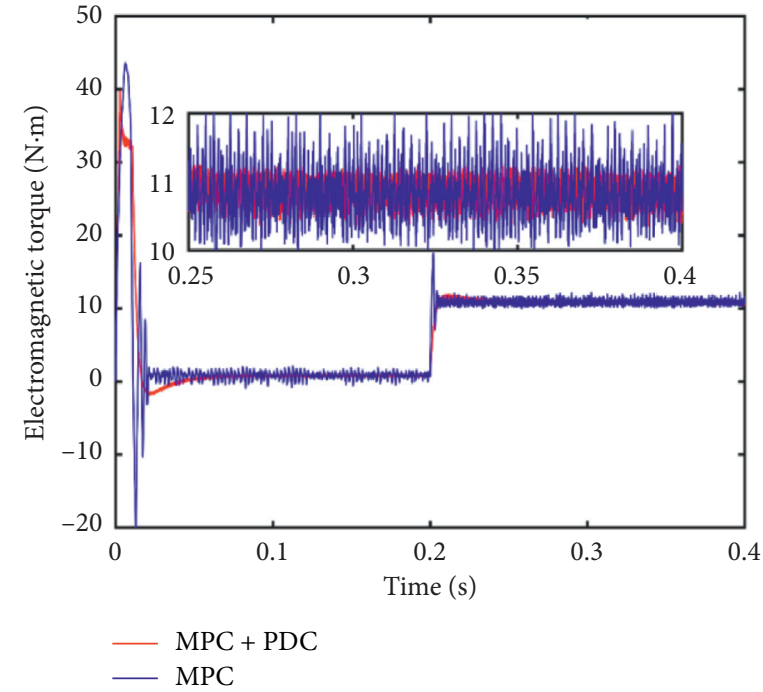

(b)

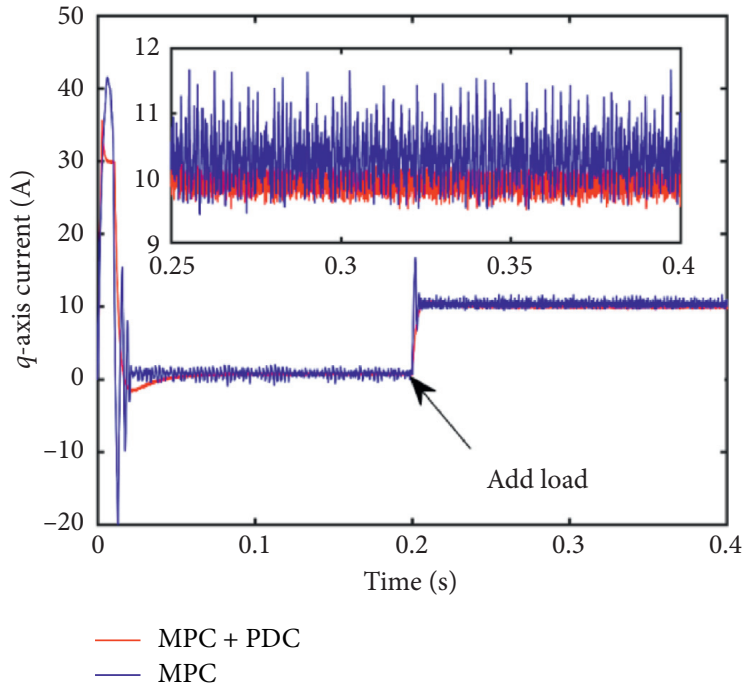

(d)

Figure 8: Comparison of MPC and MPC + PDC methods: (a) motor speed; (b) electromagnetic torque; (c) $d$-axis current; (d) $q$-axis current.

\section{Conclusion}

This paper presents MPC based on the parametric disturbance compensation method. By observing the parameter disturbance during the operation of the motor, this paper proposes a model predictive control based on parameter disturbance compensation, and through the frequency domain method, it gives the parameter tuning steps, analyzes the convergence, filtering, and parameter robustness of the model under different parameter selection states. This method has better parameter robustness and effectively avoids current static difference and oscillation problems caused by parameter changes. Under parameter changes and load conditions, the method showed strong robustness and anti-interference performance, obtained a good tracking effect, effectively reduced the speed and current ripples, and suppressed chattering.

\section{Data Availability}

The data used to support the findings of this study are included within the article.

\section{Conflicts of Interest}

The authors declare that they have no conflicts of interest.

\section{Acknowledgments}

This research was funded by the National Natural Science Foundation of China (no. 71601180).

\section{References}

[1] F. Deng and Y. Guan, "PMSM vector control based on improved ADRC," in Proceedings of the 2018 IEEE International 
Conference of Intelligent Robotic and Control Engineering (IRCE), Lanzhou, China, August 2018.

[2] X. Liu, H. Yu, J. Yu, and L. Zhao, "Combined speed and current terminal sliding mode control with nonlinear disturbance observer for PMSM drive," IEEE Access, vol. 6, pp. 29594-29601, 2018.

[3] A. K. Singh, R. Raja, T. Sebastian, and A. Ali, "Limitations of the PI control with respect to parameter variation in PMSM motor drive systems," in Proeedings of the 2019 IEEE International Electric Machines \& Drives Conference (IEMDC), San Diego, CA, USA, May 2019.

[4] J. Templos-Santos, O. Aguilar-Mejia, E. Peralta-Sanchez, and R. Sosa-Cortez, "Parameter tuning of PI control for speed regulation of a PMSM using bio-inspired algorithms," Algorithms, vol. 12, no. 3, 2019.

[5] T. Inoue, Y. Inoue, S. Morimoto, and M. Sanada, "Maximum torque per ampere control of a direct torque controlled PMSM in a stator flux linkage synchronous frame," IEEE Transactions on Industry Applications, vol. 52, no. 3, pp. 2360-2367, 2016.

[6] D. Casadei, F. Profumo, G. Serra, and A. Tani, "FOC and DTC :two viable schemes for induction motors torque control," Converter Technology \& Electric Traction, vol. 17, no. 5, pp. 779-787, 2004.

[7] L. Zhong, M. F. Rahman, W. Y. Hu, and K. W. Lim, “Analysis of direct torque control in permanent magnet synchronous motor drives," IEEE Transactions on Power Electronics, vol. 12, no. 3, pp. 528-536, 1997.

[8] Y. Zhang and J. Zhu, "Direct torque control of permanent magnet synchronous motor with reduced torque ripple and commutation frequency," IEEE Transactions on Power Electronics, vol. 26, no. 1, pp. 235-248, 2011.

[9] Y. Zhang and J. Zhu, "A novel duty cycle control strategy to reduce both torque and flux ripples for DTC of permanent magnet synchronous motor drives with switching frequency reduction," IEEE Transactions on Power Electronics, vol. 26, no. 10, pp. 3055-3067, 2011.

[10] C. He, J. Hu, and X. Ran, "Finite control set model predictive current control for PMSM based on extended state observer," in Proceedings of the 2019 14th IEEE Conference on Industrial Electronics and Applications (ICIEA), Xi'an, China, June 2019.

[11] Z. Zhang, Z. Zhang, C. Garcia, J. Rodríguez, W. Huang, and R. Kennel, "Discussion on control methods with finite-control-set concept for PMSM drives," in Proceedings of the 2019 IEEE international electric machines \& drives conference (IEMDC), San Diego, CA, USA, May 2019.

[12] X. Zhang, L. Zhang, and Y. Zhang, "Model predictive current control for PMSM drives with parameter robustness improvement," IEEE Transactions on Power Electronics, vol. 34, no. 2, pp. 1645-1657, 2018.

[13] H. A. Young, M. A. Perez, and J. Rodriguez, "Analysis of finite-control-set model predictive current control with model parameter mismatch in a three-phase inverter," IEEE Transactions on Industrial Electronics, vol. 63, no. 5, p. 1, 2016.

[14] C. Xia, M. Wang, Z. Song, and T. Liu, "Robust model predictive current control of three-phase voltage source PWM rectifier with online disturbance observation," IEEE Transactions on Industrial Informatics, vol. 8, no. 3, pp. 459-471, 2012.

[15] P. Antoniewicz and M. P. Kazmierkowski, "Virtual-flux-based predictive direct power control of AC/DC converters with online inductance estimation," IEEE Transactions on Industrial Electronics, vol. 55, no. 12, pp. 4381-4390, 2009.
[16] W. H. Chen, J. Yang, L. Guo, and S. Li, "Disturbance observerbased control and related methods: an overview," IEEE Transactions on Industrial Electronics, vol. 63, no. 2, p. 1, 2015.

[17] L. Xin and B. Z. Sensorless, "Adaptive sliding mode FCS-MPC using extended state observer for PMSM system," in Proceedings of the 2018 IEEE International Conference of Intelligent Robotic and Control Engineering (IRCE), Lanzhou, China, August 2018.

[18] Y. Zhao, X. Liu, and Q. Zhang, "Predictive speed-control algorithm based on a novel extended-state observer for PMSM drives," Applied Sciences, vol. 9, no. 12, 2019.

[19] Y.-j. Wu and G.-f. Li, "Adaptive disturbance compensation finite control set optimal control for PMSM systems based on sliding mode extended state observer," Mechanical Systems and Signal Processing, vol. 98, pp. 402-414, 2018.

[20] R. Guo, "Projective synchronization of a class of chaotic systems by dynamic feedback control method," Nonlinear Dynamics, vol. 90, pp. 53-64, 2017.

[21] R. Xu and F. Zhang, " $\epsilon$-Nash mean-field games for general linear-quadratic systems with applications," Automatica, vol. 114, Article ID 108835, 2020.

[22] W. Yao, Y. Hai Tao, G. Rong, and L. D. Yang, "Fuzzy adaptive sliding mode control of PMSM based on extended state observer," International Journal of Applied Electromagnetics and Mechanics, vol. 63, no. 3, pp. 391-407, 2020.

[23] H. Liu and S. Li, "Speed control for PMSM servo system using predictive functional control and extended state observer," IEEE Transactions on Industrial Electronics, vol. 59, no. 2, pp. 1171-1183, 2012.

[24] A. Gonzalez, V. Balaguer, P. Garcia, and A. Cuenca, "Gainscheduled predictive extended state observer for time-varying delays systems with mismatched disturbances," ISA Transactions, vol. 84, pp. 206-213, 2019.

[25] Y. Deng, J. Wang, H. Li, J. Liu, and D. Tian, “Adaptive sliding mode current control with sliding mode disturbance observer for PMSM drives," ISA Transactions, vol. 88, pp. 113-126, 2019.

[26] Y. Zhang, D. Xu, J. Liu, S. Gao, and W. Xu, "Performance improvement of model predictive current control of permanent magnet synchronous motor drives," IEEE Transactions on Industry Applications, vol. 53, no. 4, pp. 3683-3695, 2017.

[27] Y. Zhang, J. Jin, and L. Huang, "Model-free predictive current control of PMSM drives based on extended state observer using ultra-local model," IEEE Transactions on Industrial Electronics, p. 1, 2020. 